\title{
A Comparison Study of Harmonic Elimination in Cascade Multilevel Inverter Using Particle Swarm Optimization And Genetic Algorithm
}

\author{
${ }^{1}$ Rupali Mohanty, ${ }^{2}$ Sthitaprajna Rath, ${ }^{3}$ Surya Prasad Mishra \\ ${ }^{1,2,3}$ Department Of Electrical Engineering Gandhi Institute For Technology Bhubaneswar, Odisha, INDIA
}

\begin{abstract}
: so, far several methods have been presented to eliminate \& minimize the low order voltage harmonics at multilevel inverter's output \& maintain the fundamental component at the desired value. This paper focus on elimination of harmonics in a cascaded H-bridge 11 level inverter. Two heuristic techniques are implemented to find out the minimum THD in cascade multilevel inverter. Using the mathematical theory of resultants, all solutions to this equivalent problem can be found. Theoretical results are verified by simulation and experiments for an 11-level H-bridge inverter. Results show that the proposed methods effectively eliminate a great number of specific harmonics, and the output voltage is resulted in low total harmonic distortion.
\end{abstract}

Index Terms-H-bridge inverter, Harmonics, PSO, GA

\section{INTRODUCTION}

Harmonics must always be limited below threshold level prescribed by standards [1]. Several techniques have been proposed to cancel out high amplitude harmonics to eliminate or reduce the need for filtering while meeting the standard requirements. The most interesting one includes programmed harmonic elimination [2] and multilevel converters, which do not require high frequency switching as the PWM (Pulse Width Modulation) techniques do. Therefore the multilevel converters have attracted much attention in high power application. Among the different topologies for multilevel converters, the cascaded multilevel inverter has received special attention due to its modularity and simplicity of control. The principle of operation of this inverter is usually based on synthesizing the desired output voltage waveform from several steps of voltage, which is typically obtained from dc voltage sources. There are different power circuit topologies for multilevel converters. The most familiar power circuit topology for multilevel converters is based on the cascade connection of an 's' number of single-phase full-bridge inverters to generate a $(2 s+1)$ number of levels. To control the output voltage and to eliminate the undesired harmonics in multilevel converters with equal dc voltages, To control the output voltage and to eliminate the undesired harmonics in multilevel converters with equal dc voltages, various modulation methods such as sinusoidal pulse width modulation (SPWM), space vector PWM techniques are suggested in [4] and [5]. Another approach is to choose the switching angles so that specific higher order harmonics such as the 5th, 7th, 11th, and 13th are suppressed in the output voltage of the inverter. This method is known as Selective Harmonic Elimination (SHE) or programmed PWM techniques in technical literature [6]. Such method is associated with the arithmetic solution of nonlinear transcendental equations which contain trigonometric terms. This set of nonlinear equations can be solved by iterative techniques such as the Newton-Raphson method. However, such techniques need a good initial guess which should be very close to the exact solution patterns. Furthermore, this method finds only one set of solutions depending on the initial guess. Therefore, the Newton-Raphson method is not feasible to solve the SHE problem for a large number of switching angles if good initial guesses are not available.

In this paper the total harmonics are reduced by selected harmonics elimination technique in cascade multilevel inverters. In literature there are several techniques are proposed to do so. In this paper PSO and GENETIC ALGORITHM techniques are proposed to minimize the THD. In this the asymmetry of the transcendental equation set are solved and the simulation results for an 11-level cascaded multilevel inverter are discussed and a comparative analysis is done among these methods.

The paper is organized as follows - cascaded multilevel inverters are discussed in section II. The PSO \& GA techniques are discussed for minimizing the THD are given in section III \& IV. The experimental result shown in section V. The comparison between PSO \& GA technique for 11level multi inverter are shown in section VI. Section VII \& VIII followed by conclusion \& reference

\section{CASCADED MULTILEVEL INVERTER}

The cascaded H-bridge multilevel inverter consists of a series of single-phase H-bridge inverter units, as shown in Fig. 1. It is modular in nature and can be extended to any required number of levels. It is supplied from several separate dc sources (SDCSs), which may be obtained from batteries, solar cells, or ultra-capacitors. 
Each SDCS is connected to a single-phase H-bridge inverter and can generate three different voltage outputs, $+V d c, 0$, and $-V d c$. This is accomplished by connecting the dc source to the ac output side by using different combinations of the four switches $Q 1, Q 2, Q 3$, and $Q 4$. The ac outputs of the modular H-bridge inverters are connected in series such that the synthesized voltage waveform is the sum of all of the individual inverter outputs. All semiconductor devices of the H-bridges are only switching at the fundamental frequency, and consequently this is referred to as the fundamental switching scheme. Also, each H-bridge unit generates a quasi-square waveform by phase-shifting its positive and negative phase legs' switching timings. The number of output voltage levels in a cascaded multilevel inverter is then $2 s+1$, where $s$ is the number of dc sources. Threephase version of this circuit is also available by adding another two phases and connecting their neutral point together. Fig. 2 shows the generalized output voltage of cascaded H-bridge multilevel inverter with non-equal dc sources. The total output voltage is given by $v o=v 1+v 2+v 3+\ldots \ldots+v s$. With enough levels and an appropriate switching angles $\theta_{1}, \theta_{2}, \ldots \theta_{\mathrm{i}}$, and $\theta_{\mathrm{s}}$ the multilevel inverter results in an output voltage that is almost sinusoidal with a low THD with each of the active devices subjected to a single dc source, and only switching at the fundamental frequency. This reduces both the voltage stress and the switching losses of the semiconductor devices, resulting in a better utilization and high overall efficiency.

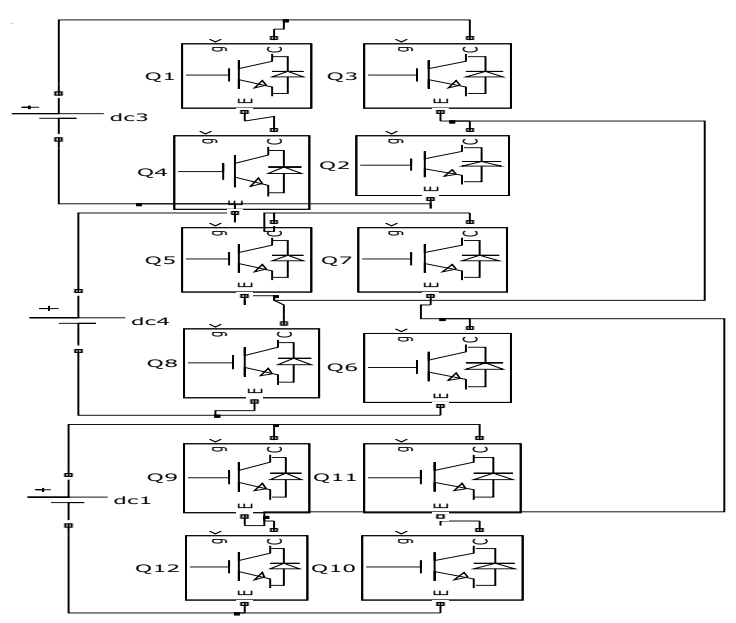

Fig 1. Cascaded H-bridge multilevel inverter

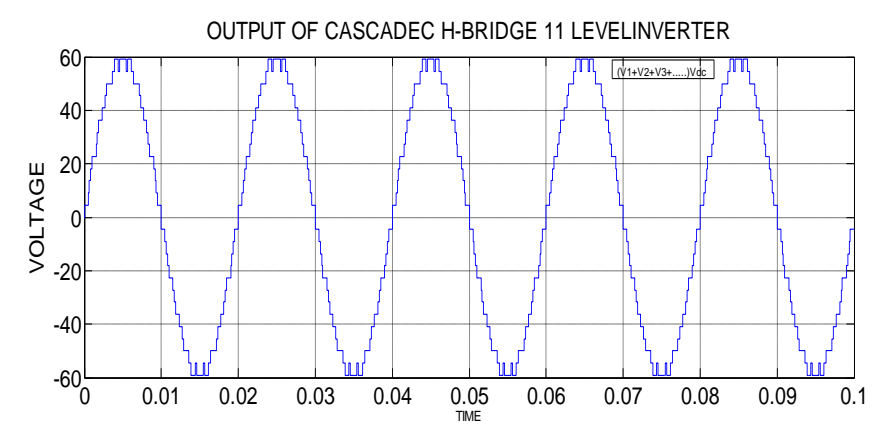

Fig 2. Output of cascaded H-bridge multilevel inverter

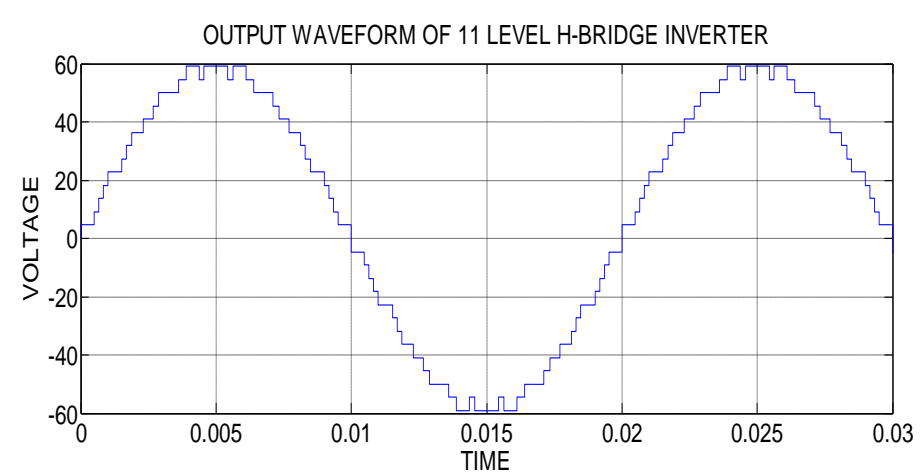

Fig3. Enlarging of cascaded H-bridge 11 level inverter 


\section{A. Problem Formulation}

Assuming the equal DC source is applied to each of the inverter and taking into consideration the characteristics of the inverter waveform Fourier series expansion of stepped output voltage waveform of the multilevel inverter with equal dc sources can be expressed as:

$V_{0} \omega t=\sum_{n=1,3,5.5}^{\infty} \frac{4 V d c}{n \pi}\left[\left\{\cos \left(n \theta_{1}\right)+\cos \left(n \theta_{2}\right)+\cos \left(n \theta_{3}\right)+\cos \left(n \theta_{4}\right)+\cos \left(n \theta_{5}\right)\right\} \sin (w t)\right]$ Where $\mathrm{V}_{\mathrm{dc}}$ is the nominal dc voltage. The above equation has 5 variables $\left(\theta_{1}, \theta_{2}, \theta_{3}, \theta_{4}, \theta_{5}\right)$ where $0<\theta_{1}<\theta_{2}<\theta_{3}<\theta_{4}<\theta_{5}<\pi / 2$. And a set of solutions is obtainable by equating s- 1 harmonics to zero and assigning a specific value to the fundamental component, as given below

$\begin{aligned} \operatorname{Cos}\left(\theta_{1}\right)+\operatorname{Cos}\left(\theta_{2}\right)+\operatorname{Cos}\left(\theta_{3}\right)+\operatorname{Cos}\left(\theta_{4}\right)+\operatorname{Cos}\left(\theta_{5}\right)=\mathrm{m} & \\ \operatorname{Cos}\left(3 \theta_{1}\right)+\operatorname{Cos}\left(3 \theta_{2}\right)+\operatorname{Cos}\left(3 \theta_{3}\right)+\operatorname{Cos}\left(3 \theta_{4}\right)+\operatorname{Cos}\left(3 \theta_{5}\right) & =0 \\ \operatorname{Cos}\left(5 \theta_{1}\right)+\operatorname{Cos}\left(5 \theta_{2}\right)+\operatorname{Cos}\left(5 \theta_{3}\right)+\operatorname{Cos}\left(5 \theta_{4}\right)+\operatorname{Cos}\left(5 \theta_{5}\right) & =0 \\ & \ldots \\ & \ldots \\ \operatorname{Cos}\left(\mathrm{n} \theta_{1}\right)+\operatorname{Cos}\left(\mathrm{n} \theta_{2}\right)+\operatorname{Cos}\left(\mathrm{n} \theta_{3}\right)+\operatorname{Cos}\left(\mathrm{n} \theta_{4}\right)+\operatorname{Cos}\left(\mathrm{n} \theta_{5}\right) & =0\end{aligned}$

Where $m=\left(\frac{V_{1}}{4 V_{d c} / \pi}\right)$ and the modulation index $m_{a}=\left(\frac{m}{s}\right)$.

For 11 level inverter where $s=5,3^{\text {rd }}, 5^{\text {th }}, 7^{\text {th }}, 9^{\text {th }}$ order harmonics will be eliminated if single phase Supply is given. In 3 phase case triple harmonics are eliminated automatically.

An objective function is then needed for the optimization procedure. In this paper the objective Function which is to be minimized is the total harmonics distortion (THD). The minimum value THD can be achieved by the optimization technique known as particle swarm optimization. The objective function is given by:

$$
F(t)=\sqrt{\sum_{n=3,5,7}^{49}\left(V_{n}\right)^{2} / V_{1}}
$$

Where $V_{1}$ is the fundamental voltage and $V n$ is the $n^{\text {th }}$ order harmonics voltage.

\section{Particle SWARM Optimization}

Optimization is the procedure of detecting attributes, configurations or parameters of a system, to produce desirable responses. Particle swarm optimization was developed by Kennedy and Eberhart (1995) as a stochastic optimization algorithm based on social simulation models. The algorithm employs a population of search points that moves stochastically in the search space. Concurrently, the best position ever attained by each individual, also called its experience, is retained in memory. This experience is then communicated to part or the whole population, biasing its movement towards the most promising regions detected so far. The communication scheme is determined by a fixed or adaptive social network that plays a crucial role on the convergence properties of the algorithm. The development of particle swarm optimization was based on concepts and rules that govern socially organized populations in nature, such as bird flocks, fish schools, and animal herds

\section{A. PSO algorithm for minimizing THD}

: Let $\theta_{i}=\left[\theta_{i l}, \theta_{i 2}, \ldots, \theta_{i s}\right]$ be a trial vector representing the $i_{\text {th }}$ particle of the swarm to be evolved. The elements of $\theta_{i}$ are the solutions of the harmonic minimization problem, and the $d$ th element of that is corresponding to the $d t h$ switching angle of the inverter. The step-by-step procedure to solve the SHE problem with equal de sources is as follows.

1) Get the data for the system. At the first step, the required parameters of the algorithm such as population size $M$, maximum iteration number iter mix, etc., are determined and the iteration counter is set to iter $=1$.

2) Generate the initial conditions of each particle. Each particle in the population is randomly initialized between 0 and $\pi / 2$; similarly, the velocity vector of each particle has to be generated randomly within $-V \max$ and $V \max$.

3) Evaluate the particles. Each particle is evaluated using the fitness function of the harmonic minimization problem. the cost function is given as follows

$$
F(t)=\sqrt{\sum_{n=3,5,7}^{49}\left(V_{n}\right)^{2} / V_{1}}
$$

4) Update the personal best position of the particles. If the current position of the $i$ th particle is better than its previous personal best position, replace $P i$ with the current position $X i$. In addition, if the best position of 
the personal bests of the particles is better than the position of the global best, replace $P g$ with the best position of the personal bests.

5) Update the velocity and vectors. All particles in the population are updated by velocity and position update rules (4) and (5), respectively.

6) Termination criteria. If the iteration counter iter reaches itermax, stop; else, increase the iteration counter iter $=$ iter +1 and go back to $\operatorname{step}(3)$.

Continuing by an example, let us consider a five-level inverter for simplicity, which includes the series connection of two H-bridge inverters. For this case, there are two SDCSs (one for each bridge), the values of which are generally equal.

\section{GENETIC ALGORITHM}

Genetic algorithm is a directed search algorithms based on the mechanics of biological evolution. It was developed by John Holland, University of Michigan in 1970.It is based on "Darwin's Theory of Evolution" In the computer science field of artificial intelligence, a genetic algorithm (GA) is a search heuristic that mimics the process of natural evolution. This heuristic is routinely used to generate useful solutions to optimization and search problems. Genetic algorithms belong to the larger class of evolutionary algorithms (EA), which generate solutions to optimization problems using techniques inspired by natural evolution, such as inheritance, mutation, selection, and crossover.

\section{A. Genetic Algorithm for minimizing the THD:}

1. Randomly initialize the chromosome size and then initialize the iteration size.

2. Find out the objective value (THD) \& convert the decimal no. to binary no.

3. Now cross-over between different sets of population.

4. Those chromosomes that are less fittest remove that set of chromosome and initialize new chromosome on that place.

5. If maximum iteration reached then terminate or go back to step 3.

\section{A. For Particle Swarm Optimization}

\section{EXPERIMENTAL RESULT}

In order to validate the computational results as well as the

simulations, experimental results are presented for a single phase 11-level cascaded H-bridge inverter .The program was developed in mat lab and the cost function i.e. the THD was minimized. The 'Gbest' means at which switching angle the minimum THD has come how found out. THD result of the output line voltage up to $49^{\text {th }}$ harmonics was calculated. for this case the THD result was $6 \% .10$ volts dc supply was given to the inverters. The switching angle at which this result has come is as follows

$\theta_{1}=5.2338, \theta_{2}=16.3852, \theta_{3}=30.9033, \theta_{4}=42.9065$,

$\theta_{5}=62.6564$ in degree.

The Fourier transform(FFT) analysis has done and the figure shown below,

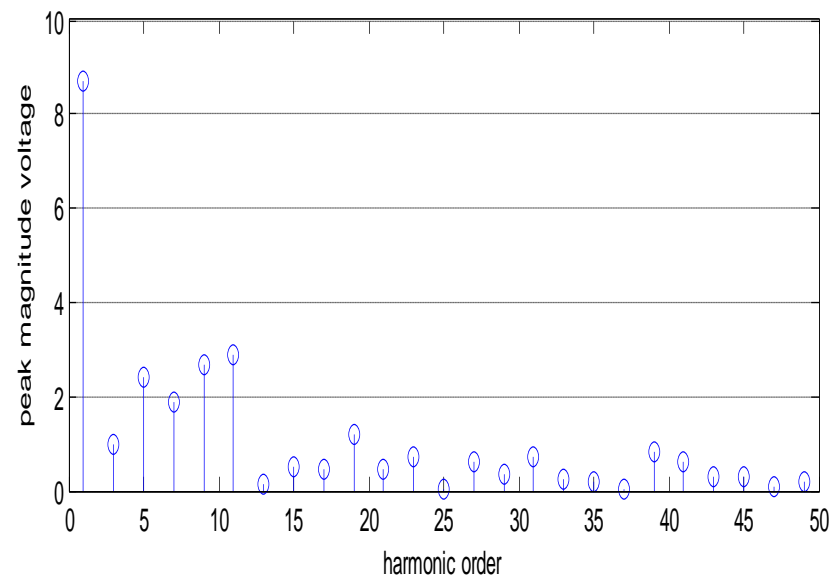

Fig 4. FFT analysis for modulation index 0.76 


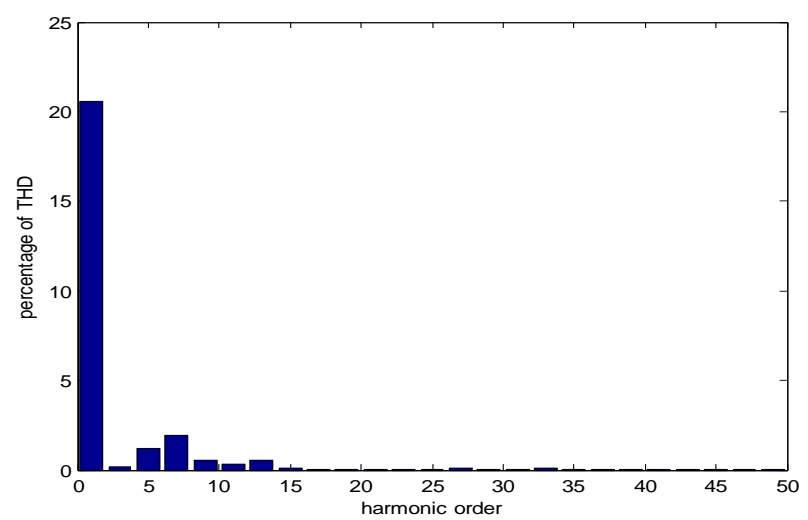

Fig 5. FFT analysis with percentage of THD

\section{B. For Genetic Algorithm}

We are using the genetic algorithm in our Matlab Programming in order to obtain the best best firing angle for minimum THD. THD result of the output line voltage up to $49^{\text {th }}$ harmonics was calculated. for this case the THD result was $6.83 \%$. 10 volts dc supply was given to the inverters. The switching angle at which this result has come is as follows

$\theta_{1}=1, \theta_{2}=2, \theta_{3}=5, \theta_{4}=6, \theta_{5}=16$.in degree.

The Fourier transform(FFT) analysis has done and the figure shown below

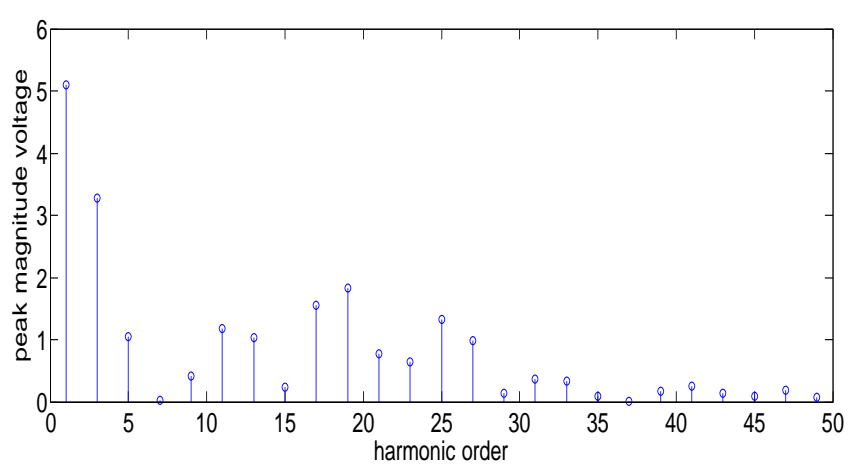

Fig 6. FFT analysis for modulation index 0.8

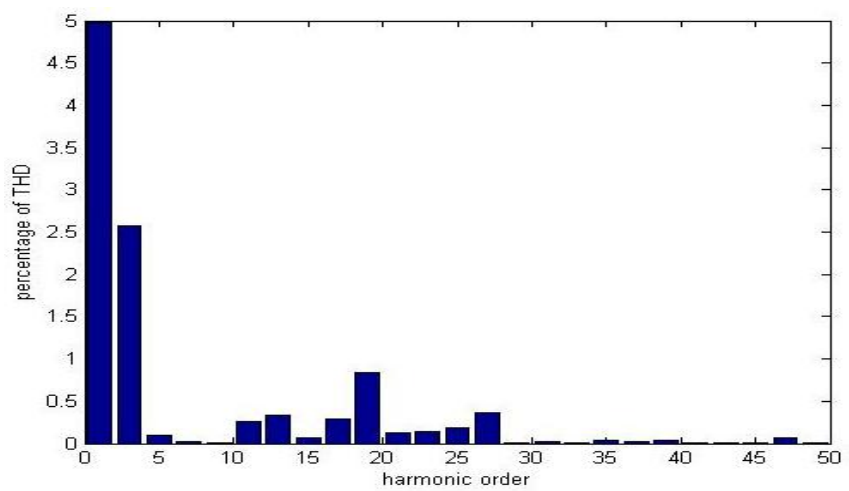

Fig 7. FFT analysis with percentage of THD 
VI.

SIMULATION RESULT

A. Output voltage waveform by using PSO

OUTPUT VOLTAGE WAVEFORM BY USING PSO

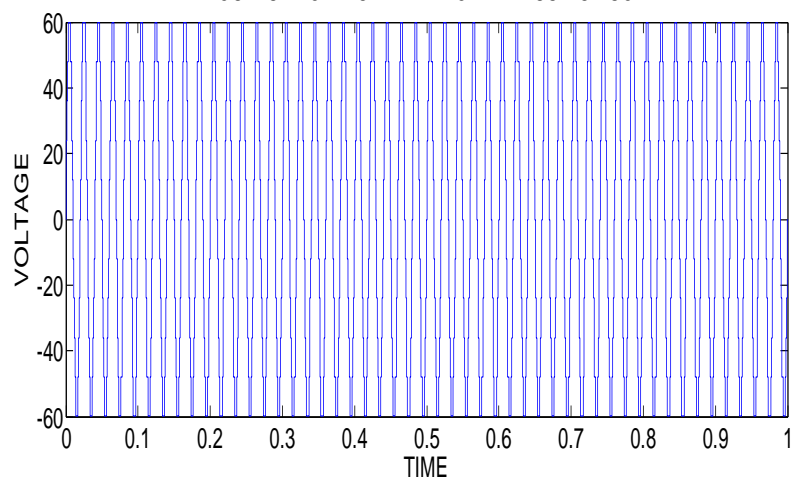

Fig 8. Output voltage wave form 11 level inverter using PSO technique OUTPUT VOLTAGE WAVEFORM USING PSO

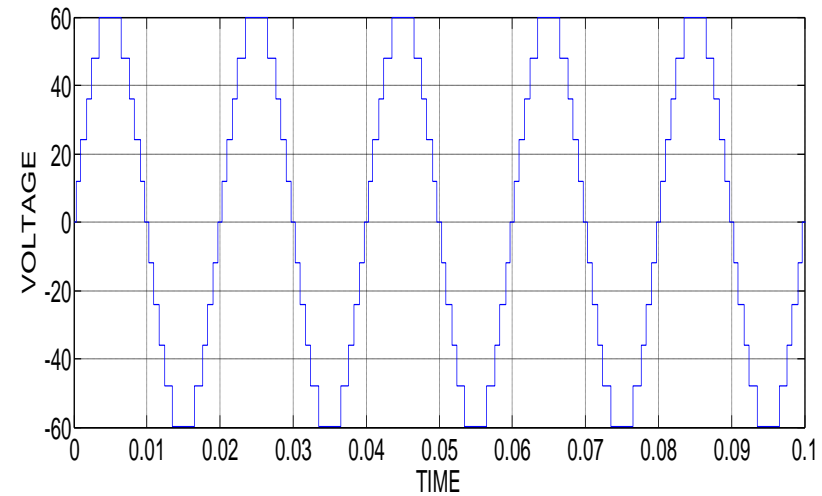

Fig 9.enlarging Output voltage waveform 11 level inverter for PSO

B. Output voltage waveform by using $G A$

OUTPUT VOLATEG WAVEFORM USING GA TECHNIQUE

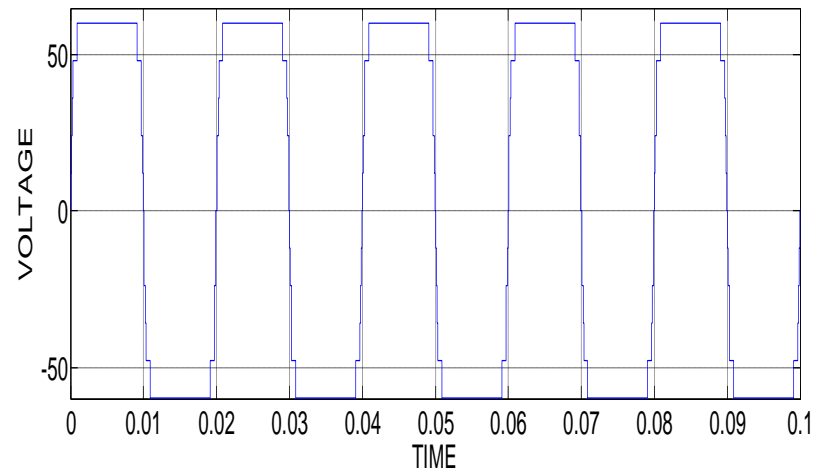

Fig10. Output voltage waveform 11 level inverter for $G A$

C. Comparative Statement

TABLE I. COMPARATIVE STATEMENT BETWEEN PSO \&GA

\begin{tabular}{|l|l|}
\hline \multicolumn{1}{|c|}{ PSO } & \multicolumn{1}{|c|}{ GA } \\
\hline THD $=6.0 \%$ & THD $=6.8 \%$ \\
\hline THDfor $3^{\text {rd }}$ harmonic $=0.5 \%$ & THDfor $3^{\text {rd }}$ harmonic $=2 \%$ \\
\hline THD for $5^{\text {th }}$ harmonic $=2 \%$ & THD for $5^{\text {th }}$ harmonic $=0.1 \%$ \\
\hline $\begin{array}{l}\text { Out put voltage waveform } \\
\text { Is very smooth }\end{array}$ & $\begin{array}{l}\text { Out put voltage waveform } \\
\text { Is less smooth }\end{array}$ \\
\hline $\begin{array}{l}\text { It can apply for same as well } \\
\text { as different source of voltage }\end{array}$ & It can apply for same voltage source \\
\hline $\begin{array}{l}\text { Run time of the program is } \\
\text { very less }\end{array}$ & $\begin{array}{l}\text { Run time of the program is more than } \\
\text { that of PSO }\end{array}$ \\
\hline
\end{tabular}




\section{CONCLUSION}

The prime objective of this project is to develop programs on different heuristic technique to solve the SHE problem with equal D.C sources in H-bridge cascade multilevel inverter. The PSO and GA techniques presented in this thesis achieve this objective and includes:

1. Development of algorithm for minimization of THD .

2. Application of this algorithm in multilevel inverters with equal dc sources which are used in power system to convert the de power to ac power.

3. Development of simulation to validate the result.This concludes that when the resultant approach reaches the limitation of contemporary algebra software tools, the proposed methods are able to find the optimum switching angles in a simple manner. We found that by developing logics for the problem, the optimum solution is found out. These methods are used less equations, less derivations and took less time than that of the other methods of harmonic elimination discussed in chapter to solve the problem. The convergence criteria are very smooth. The simulation and experimental results are provided for an 11-level cascaded H-bridge inverter to validate the accuracy of the computational results. From the experiment we found that the percentage of THD is more in GA technique than that of PSO technique.

\section{REFERENCE}

[1]. IEEE recommended practices and requirements for harmonic control in electrical power system IEEE standard,519-1992.

[2]. M. Sarvi, M. R. Salimian, "Optimization of Specific Harmonics in Multilevel Converters by GA \& PSO”,UPEC2010 31 st Aug - 3rd Sept 2010

[3]. H. Taghizadeh and M. Tarafdar Hagh,"Harmonic Elimination of Cascade Multilevel Inverters with Nonequal DC Sources Using Particle Swarm Optimization" IEEE transactions on industrial electronics, vol. 57, no. 11, november 2010.

[4]. D. G. Holmes and T. A. Lipo, "Pulse Width Modulation for Power Converters". Piscataway, NJ: IEEE Press, 2003

[5]. S. Kouro, J. Rebolledo, and J. Rodriguez, "Reduced switching-frequency modulation algorithm for high Power multilevel inverters," IEEE Trans.Ind. Electron., vol. 54, no.5, pp. 2894-2901, Oct. 2007

[6]. W. Fei, X. Du, and B. Wu, "A generalized half-wave symmetry SHE-PWM formulation for multilevel voltage inverters," IEEE Trans.Ind. Electron., vol. 57, no. 9, pp. 3030- 3038, Sep. 2010

[7]. Leon M. Tolbert, John N. Chiasson, Zhong Du, Keith J. McKenzie, "Elimination of Harmonics in a

[8]. Multilevel Converter",IEEE transactions on application industry,vol.41,n0.1,January/februry 2005

[9]. Jagdish Kumar,et all "Selective Harmonic Elimination Technique for a Multilevel Inverter", Fifteenth National Power Systems Conference (NPSC), IIT Bombay, December 2008

[10]. Burak Ozpineci, Leon M. Tolbert'.2, John N. Chiasson2, "Harmonic Optimization of Multilevel Converters Using Genetic Algorithms", 2004 35th Annul IEEE Power Electronics Specialisu Conference.

\section{AUTHOR PROFILES}

1) RUPALI MOHANTY is a faculty member in the Department of electrical engineering, Gandhi Institute for Technology. She received her M.TECH degree in power electronics \& drive from the KIIT University, Bhubaneswar in 2012. Her main professional interests are in power electronics, electrical drive.

2) STHITAPRAJNA RATH is a faculty member in the Department of electrical engineering, Krupajala Engineering College. He received his M.TECH degree in power energy \& system from KIIT University, Bhubaneswar in 2013. His main professional interests are in power system operation \& control, control system engineering, renewable energy system and mathematical modeling

3) SURYA PRASAD MISHRA is a faculty member in the Department of electrical engineering, Gandhi Institute for Technology. He received her M.TECH degree in power system engineering from the BijuPattnaik University, Bhubaneswar in 2013. His main professional interests are in control system engineering, electrical machine, power electronics and drive 